\title{
A virtual laboratory system for physiology teaching
}

\author{
Geoff Brindle*, William Mitchell, Len Seal** and Karl Bohme** \\ Manchester Metropolitan University; "Department of Computing; \\ **Department of Biological Sciences
}

The problem of understanding physiological processes can be aided by visualization tools. Traditionally this has been achieved by the use of schematic paper diagrams. However, many physiological processes are quite complex, and in many instances students encounter difficulties in understanding the dynamics. This paper describes the rationale behind an alternative approach using interactive three-dimensional computer-graphics simulation to aid comprehension of scientific concepts.

\section{Introduction}

There exist a number of areas in the teaching of physiology which potentially lend themselves to a Computer-Based Learning approach. One such area which has been explored at Manchester Metropolitan University (MMU) and elsewhere (Dewhurst, 1993; Kwan, 1993) is the use of multimedia tools to simulate aspects of experiments traditionally performed on animals. The use of real animal specimens (for example, frogs or rats) for dissection and experimentation is both costly and contrary to the ethics of some students.

A related but different area which we are currently investigating is that of scientific visualization in relation to animal physiology - that is, the visualization of processes which often are difficult or impossible to see except either through the measurements generated on instruments such as chart recorders and oscilloscopes, or the diagrams provided by textbooks. This information is generally of an abstract nature, and students find some of it difficult to grasp using two-dimensional static diagrams of the kind provided in textbooks or handouts. The nature of the textbook medium prohibits some key dynamic ideas coming across. At MMU a collaborative study between the departments of Computing and Biological Sciences is investigating the possibility of using three-dimensional interactive simulations, or Virtual Laboratory environments, to 
provide concept visualization tools for biologists. The present study is focused on one particular set of physiological processes - those which occur at the neuromuscular junction. That process, it is hoped, will provide a vehicle for exploring the requirements of related applications and lead to the development of software tools which can be used to generate solutions more generally.

\section{The neuromuscular junction}

The junction between nerve strands and muscle fibres is typical of a number of physiological processes where understanding is difficult using standard diagrams. The sequence of events which occurs at the neuromuscular junction during the initiation of muscle contraction is complex. It includes the arrival of action potentials, calcium flux changes, merging of synaptic vesicles with junction membrane, release of neurotransmitter from vesicles into the junction cleft, interaction with muscle membrane receptors, pore changes and ion fluxes. An appreciation of the dynamic aspects of the sequence is essential to an understanding of the varying levels of activity seen in muscle ranging from a single twitch to a sustained contraction. A clear understanding of the basic process is also essential before consideration of variation in activity at these junctions associated with the effects of drugs, toxins and disease processes.

\section{The Virtual Reality approach}

There are many variants of Virtual Reality (VR) systems. A good overview is provided by Barker in an earlier issue of $A L T-J$ (Barker, 1993).

VR systems are less of a new technology than an integration of several existing technologies, the chief of which are 3D computer graphics display and animation, computer modelling and simulation, real-time systems and novel input and output device technologies. When these technologies are combined fully, it is possible to produce systems where the user can feel immersed within an authentic, albeit synthetic world and where he or she can manipulate that world in a natural and familiar way. In such a system, the user interface becomes transparent, and the user feels as though he/she is inside the model data space. Rheingold (1991) provides a very readable and detailed historical perspective of VR technologies.

\section{Advantages of a VR approach}

The underlying assumption of this project is that the construction and use of systems can be guided by the VR ideal even though they may fall somewhat short of a fully immersive system. Some of the reasons for such a stance are:

- The use of a 3D model and 3D viewing facilities allow more complex objects, behaviours and relationships to be displayed and manipulated. This is important where the relationships in the world being modelled are complex. The value of $3 \mathrm{D}$ representations has been repeatedly shown in the field of Computer Aided Design (CAD). 
- VR systems go beyond traditional CAD modelling in the degree of realism provided. Texture is added to the surface of object geometries to provide detail, and the use of perspective and illumination/reflection modelling provides depth cues. This gives a sense of authenticity so that the user can use intuitive notions of depth and space derived from everyday life to understand the structures and relationships in the conceptual world.

- VR provides simulation based on a stored model of the process under study so that the parameters of this model can be changed. Understanding can be actively acquired by experimentation, a method in keeping with current educational thinking.

- A key feature of virtual worlds is interactivity. It should be possible to interact with synthetic worlds in the same way as we interact with real worlds. As a result, interaction tasks are more natural, and the amount of training needed is reduced (Balaguer \& Gobbetti, 1993).

- Scientists are trained in the use of instruments and measuring devices as well as in the specifics of the particular process or object under consideration. Typically, students need to learn how to calibrate instruments, how to position measurement probes, and how to use the options and switches on instrument panels. If learning how to use the computer interface can be made identical to learning to use traditional instrument interfaces, then existing knowledge of such interfaces which the student brings to the interaction can be profitably used and reinforced.

\section{A prototype virtual laboratory}

A prototype virtual laboratory is currently at an early stage of development at MMU. For development purposes, a VR development system - World Tool Kit for Windows provides a set of rendering tools and interaction controls which are called from $\mathrm{C}$ programming language interface. The software runs on a PC but requires good floatingpoint capabilities (DX2 or Pentium) processors. Since the intention is to use the system with undergraduate students, it is important that it can be implemented on a desktop system using relatively cheap interaction equipment.

\section{Some areas of difficulty}

The VR laboratory is clearly an ambitious project offering many potential problems and challenges. While initial work has been promising, we are aware of a number of key problem areas:

\section{Animation}

Desktop VR development environments are proving to be usable in a number of contexts. However, animation is usually in the form of a walkthrough or flyby where the world being navigated stays relatively static. The provision of worlds containing multiple interacting objects is more problematic. Convincing animation requires attention to many details concerning the way that objects move, and it is traditionally a time-consuming programming activity. 
Interaction

The design and development of virtual tools is an area requiring substantial design and development effort. Researchers are only just beginning to explore the possibilities (Balaguer \& Gobetti, 1993).

Development lead times

It is clear that the provision of authoring tools is ultimately necessary if technologies like VR are to have any impact on learning. The amount of work involved in producing learning material is otherwise excessive and the need for specialist subject knowledge is a limitation. It should be possible for lecturers in a particular specialism to produce interactive simulations themselves easily, and within a reasonable timescale (Van Dam, 1992).

\section{Conclusions}

The virtual-world ideal, while perhaps not perfectly realizable given today's technology and the need for desktop solutions, can nevertheless be used as a model to guide the design of CAL tools. A range of VR development toolkits and authoring systems are beginning to emerge, and these will be added to as more experience is gained. Already these tools are being used in entertainment and in the design of products in industry. At MMU we are developing tools aimed at the application of VR in specific educational contexts, with the aim of providing software tools that can be used generally in the area of conceptual visualization.

\section{References}

Balaguer, J.F. \& Gobbetti, E. (1993), 'Virtuality Builder II: on the topic of 3D interaction' in Thalmann, N.M. \& Thalmann, D. (eds), Virtual Worlds and Multimedia, Chichester, Wiley.

Barker, P. (1993), 'Virtual Reality: theoretical basis, practical applications', Association for Learning Technology Journal, 1, 1, 15-25.

Dewhurst, D.G. \& Williams, A. (1993), 'Frog skin: a computer simulation of experiments performed on frog skin in vitro to investigate the epithelial transport of ions', $A T L A, 21$, $3,350-8$.

Kwan, T.K. (1993), A Multimedia Learning Environment for Dissection Skills in Biology, M.Sc. dissertation, Sheffield University.

Rheingold, H. (1991), Virtual Reality, London, Secker \& Warburg.

Van Dam, A. (1992), 'Electronic books and interactive illustrations' in Cunningham, S. \& Hubbold, R.J. (eds), Interactive Learning Through Visualisation, Berlin, Springer-Verlag. 